\title{
Patterns of care analysis for salivary gland cancer: a survey within the German Society of Radiation Oncology (DEGRO) and recommendations for daily practice
}

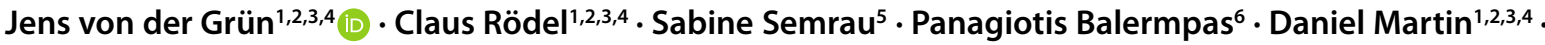 \\ Rainer Fietkau ${ }^{5}$. Marlen Haderlein ${ }^{5}$
}

Received: 27 November 2020 / Accepted: 16 July 2021 / Published online: 24 August 2021

(c) The Author(s) 2021

\begin{abstract}
Background Salivary gland cancer (SGC) is rare and a heterogeneous type of cancer. Prospective randomized trials are lacking. No guideline focusing on standard procedures of radiotherapy (RT) in the treatment of SGC exists. Therefore, we surveyed the members of the German Society of Radiation Oncology (DEGRO) to gain information about current therapeutic strategies of SGC.

Methods An anonymous questionnaire was designed and made available on the online platform umfrageonline.com. The corresponding link was sent to all DEGRO members who provided their user data for contact purposes. Alternatively, a PDF printout version was sent. Frequency distributions of responses for each question were calculated. The data were also analyzed by type of institution.

Results Sixty-seven responses were received, including answers from 21 university departments, 22 non-university institutions, and 24 radiation oncology practices. Six participants reported that their departments (practice: $n=5$, non-university hospital: $n=1$ ) did not treat SGC, and therefore the questionnaire was not completed. Concerning radiation techniques, target volume definition, and concomitant chemotherapy, treatment strategies varied greatly among the participants. Comparing university vs. non-university institutions, university hospitals treat significantly more patients with SGC per year and initiated more molecular pathological diagnostics.

Conclusion SGC represents a major challenge for clinicians, as reflected by the inhomogeneous survey results regarding diagnostics, RT approaches, and systemic therapy. Future prospective, multicenter clinical trials are warranted to improve and homogenize treatment of SGC and to individualize treatment according to histologic subtypes and risk factors.
\end{abstract}

Keywords Salivary gland cancer $\cdot$ Molecular diagnostics $\cdot$ Systemic therapy $\cdot$ Radiotherapy $\cdot$ Stereotactic ablative body radiotherapy

Availability of data and material The datasets generated and/or analyzed during the current study are available from the corresponding author upon reasonable request.

$\triangle$ Jens von der Grün, MD

jens.vondergruen@kgu.de

1 Department of Radiotherapy and Oncology, University Hospital Frankfurt, Goethe University, Frankfurt am Main, Germany

2 German Cancer Research Center (DKFZ), Heidelberg, Germany
3 German Cancer Consortium (DKTK), Partner Site Frankfurt am Main/Mainz, Frankfurt am Main, Germany

4 Frankfurt Cancer Institute (FCI), Goethe University, Frankfurt am Main, Germany

5 Department of Radiation Oncology, University Hospital of Erlangen, Friedrich Alexander University of Erlangen-Nuremberg, Erlangen, Germany

6 Department of Radiation Oncology, University Hospital Zurich, Zurich, Switzerland 
Fig. 1 Professional environment of the participants and annual case numbers; a professional environment of the participants; b distribution of annually treated salivary gland cancer patients reported by participants

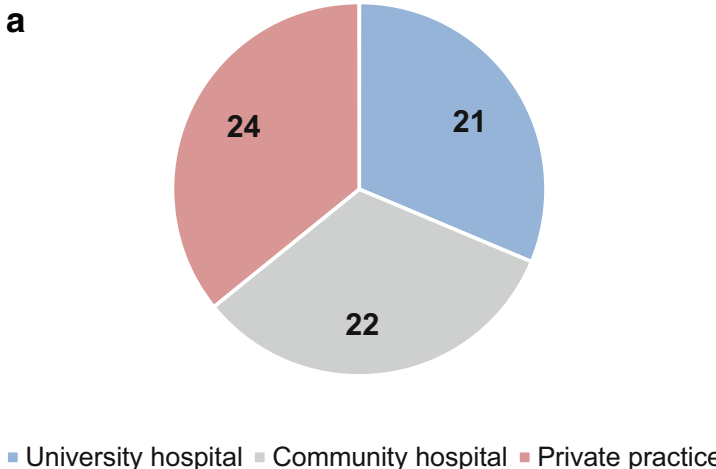

- University hospital $₫$ Community hospital $₫$ Private practice

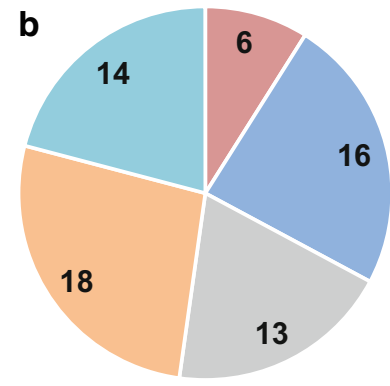

$\| 0=1-3=3-5-5-10 \quad->10$

\section{Introduction}

Salivary gland cancer (SGC) is a rare tumor entity including a variety of different histologic subtypes. Due to the lack of prospective, randomized trials, therapeutic strategies remain controversial and no general guideline focusing on detailed recommendations for radiotherapy (RT) in SGC exists. Treatment recommendations are usually based on retrospective data. Surgery, if possible, is the primary treatment of SGC. Furthermore, large retrospective studies indicated a benefit of postoperative external beam radiotherapy (PORT) in locally advanced and/or high-grade SGC $[1,2]$. In patients with adenoid cystic carcinomas (AdCC), high linear energy transfer (LET) radiation with protons or carbon ions might be beneficial in case of macroscopic residual or inoperable disease [3]. However, many details on diagnostics and treatment of SGC remain unclear, such as target volume definition, dose prescription, treatment of metastatic disease, and the role of systemic therapy. We thus surveyed the members of the German Society of Radiation Oncology (DEGRO) to gain information about reallife concepts regarding diagnostics and RT for SGC.

\section{Materials and methods}

A pattern of care questionnaire (supplementary table 1) assessing diagnostic and treatment modalities of SGC in radiation oncology departments was developed. The questionnaire focused on general information on participating institutions, indications, diagnostic procedures, target volume definition, RT techniques, and concomitant chemotherapy (CTX). Moreover, five case reports with a total of 18 questions were queried. The questionnaire was reviewed by all listed authors and was made available on the online platform umfrageonline.com. The corresponding link was sent to all DEGRO members who provided their user data for contact purposes. Alternatively, a printout PDF copy was sent. The online survey was available from May 5 to August 23, 2020. Within that period, a total of three reminder e-mails were sent. SPSS (IBM SPSS Statistics, v24.0, Armonk, NY, USA) was used for analysis. Frequency distributions of responses for each question were calculated. The data were further analyzed by type of institution (university department vs. non-university institution/outpatient practice) using Pearson's chi-squared test. Statistical significance was considered at $p \leq 0.05$.

\section{Results}

\section{General information on diagnostics and treatment of SGC}

Sixty-seven responses were received, including answers from 21 university departments, 22 non-university departments, and 24 radiation oncology practices (Fig. 1a). Six participants reported that their department (practice: $n=5$, non-university hospital: $n=1$ ) did not treat SGC, and therefore the questionnaires were not completed and not considered in the following evaluation. The reported case numbers of the participants are shown in Fig. 1b.

The following annual SGC case numbers were reported ( $n=61): 26.2 \%$ of the participants treat $1-3$ patients, $21.3 \%$ 3-5 patients, $29.5 \%$ 5-10 patients, and 23\% >10 patients per year. In $59(96.7 \%)$ of the institutions, head and neck

Table 1 Numbers of centers routinely using additional molecular pathological diagnostics in salivary gland cancer

\begin{tabular}{lll}
\hline Molecular pathological diagnostics & $n$ & $\%$ \\
\hline HER2neu amplification & 33 & 54.1 \\
Androgen receptor & 15 & 24.6 \\
Estrogen receptor & 11 & 18.0 \\
Progesterone receptor & 11 & 18.0 \\
PD-L1 status & 23 & 37.7 \\
TRK fusion & 12 & 19.7 \\
RET fusion & 6 & 9.8 \\
Next-generation sequencing/multi gene panels & 6 & 9.8 \\
\hline
\end{tabular}

$P D-L 1$ programmed death-ligand $1, T R K$ tropomyosin receptor kinase, $R E T$ ret proto-oncogene 
Fig. 2 Radiation techniques and indications for nerve tract irradiation. a Preferred radiation technique according to tumor subtype and treatment situation, b indications for radiotherapy of nerve tracts up to the base of skull. $R T$ Radiotherapy, $P O R T$ postoperative radiotherapy, IMRT intensity-modulated radiotherapy, AdCC adenoid cystic carcinoma, VMAT volumetric arc therapy, $S G C$ salivary gland cancer a

Radiation techniques (\%)

\begin{tabular}{|c|c|c|c|c|c|}
\hline AdCC, primary RT & 44 & 7 & 43 & & \\
\hline AdCC, PORT & 10 & 70 & 2 & 18 & \\
\hline non-AdCC, primary RT & 10 & 79 & & 22 & 8 \\
\hline \multirow[t]{2}{*}{ non-AdCC, PORT } & 10 & 85 & & & 23 \\
\hline & $\begin{array}{l}\text { IMRT } \\
\text { Rotational IMRT (VMA } \\
\text { Treatment with proton } \\
\text { Treatment with carbon } \\
\text { Referral to carbon ion/ }\end{array}$ & $\begin{array}{l}\text { arc) } \\
\text { nerapy ce }\end{array}$ & & & \\
\hline
\end{tabular}

b RT of nerve tracts up to the base of skull (\%) cancer patients are routinely discussed in an interdisciplinary tumor board. A reference pathological second opinion for SGC is gathered in 13 (21.3\%) of the participating centers and in $34(55.7 \%)$ of the participating centers, further molecular pathological diagnostics are available (Table 1).

The most common radiotherapy concept was PORT $(n=57,93.4 \%)$, followed by primary $(n=3,4.9 \%)$, and palliative RT $(n=1,1.6 \%)$. For definitive RT treatment planning, the participants recommended the following imaging modalities: computed tomography $(\mathrm{CT})$ with contrast agent: $n=47$ (77\%); magnetic resonance imaging (MRI) with contrast agent: $n=53$ (86.9\%); fluorodeoxyglucose positronemission tomography (FDG-PET) CT: $n=10$ (16.4\%); choline/prostate-specific membrane antigen (PSMA) PETCT: $n=1(1.6 \%)$. For PORT treatment planning the following diagnostic procedures were recommended: preoperative CT with contrast agent: $n=46(75.4 \%)$; preoperative MRI with contrast agent: $n=48$ (78.7\%); postoperative CT with contrast agent: $n=28$ (45.9\%); postoperative MRI with contrast agent: $n=26(42.6 \%)$; preoperative FDG-PETCT: $n=5(8.2 \%)$. The preferred treatment technique as re-

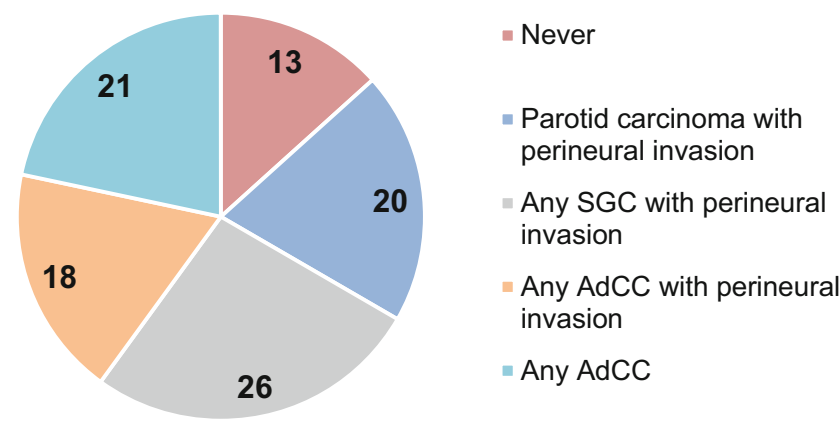

ported by participants was intensity-modulated RT (IMRT). However, the distribution of the preferred treatment technique varies according to tumor subtype (adenoid cystic vs. non-adenoid cystic) and treatment concept (definitive vs. postoperative; Fig. 2a).

RT of the nerve pathways up to the base of the skull is performed as follows: never: $n=8(13.1 \%)$; in case of parotid carcinoma with perineural invasion: $n=12$ $(19.7 \%)$; in case of all SGC with perineural invasion: $n=16(26.2 \%)$; in case of all AdCC with perineural invasion: $n=11(18.0 \%)$; and in case of all AdCC: $n=13$ (21.3\%; Fig. 2b).

Of the participants, $21.3 \%(n=13)$ never apply concomitant CTX/systemic therapy in patients with SGC in the definitive situation and $39.3 \%(n=24)$ in the postoperative situation. Indications for CTX/systemic therapy, when recommended by participants, are listed in Table 2 .

Chosen systemic therapy regimes were distributed as follows: platinum-based: $n=37$; platinum- and 5-FU: $n=11$; cetuximab: $n=1$; targeted therapies: $n=1$. 
Table 2 Indications for concomitant chemotherapy in the treatment of salivary gland cancer

\begin{tabular}{lll}
\hline Indication for chemotherapy & $\begin{array}{l}\text { Definitive treatment } \\
\text { situation }\end{array}$ & $\begin{array}{l}\text { Postoperative treatment } \\
\text { situation }\end{array}$ \\
\hline $\begin{array}{l}\text { For all high-risk salivary gland cancers according to recommendations for squamous } \\
\text { cell carcinomas of the head and neck region }\end{array}$ & $15(27 \%)$ & $10(24 \%)$ \\
$\begin{array}{l}\text { For all kinds of salivary gland cancers according to recommendations for squamous } \\
\text { cell carcinomas of the head and neck region }\end{array}$ & $9(16 \%)$ & $4(10 \%)$ \\
According to individual decision & $31(56 \%)$ & $28(67 \%)$ \\
\hline
\end{tabular}

\section{Case studies}

Cases 1 to 3 described two cases of AdCC and one of salivary duct carcinoma (SDC) in the postoperative situation. Detailed case descriptions can be found in supplementary table 1 and results from the survey in Fig. 3. With increasing risk, the number of institutions applying combined chemoradiotherapy (CRT) increases (see Fig. 3e). In case $3,77 \%$ of the participating institutions would apply systemic therapy: 34 institutions would apply simultaneous chemotherapy, 8 institutions simultaneous trastuzumabbased systemic therapy, and 5 institutions trastuzumabbased systemic therapy after radiotherapy.

Case 4 presented a patient with low-grade acinic cell carcinoma (AciCC) with the following tumor status: pT4 cN0 cM0 R1; second resection not possible or refused by patient. In this case, $55.7 \%$ of the participants indicated PORT, 24.6\% postoperative chemoradiotherapy (POCRT), $14.8 \%$ watch-and-wait, and $4.9 \%$ did not answer the question. a

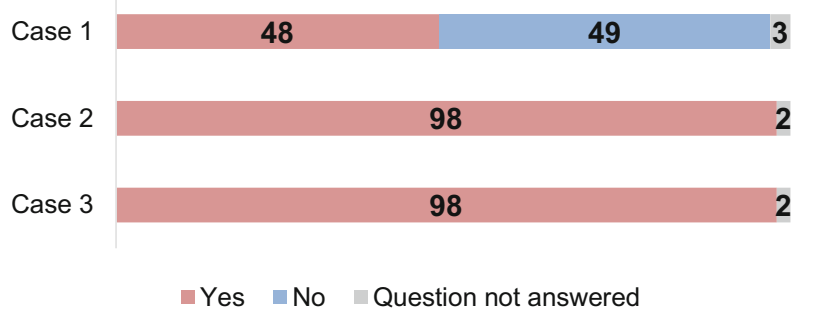

C

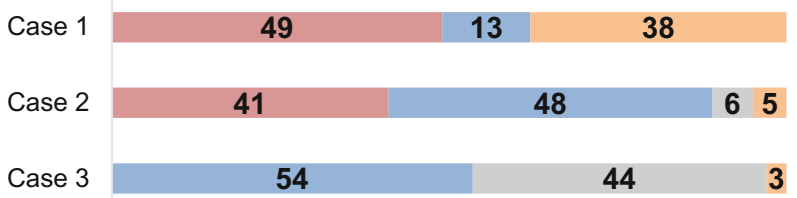

b

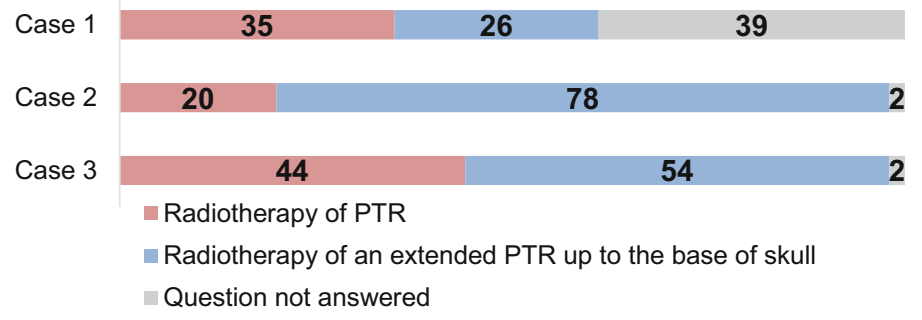

d

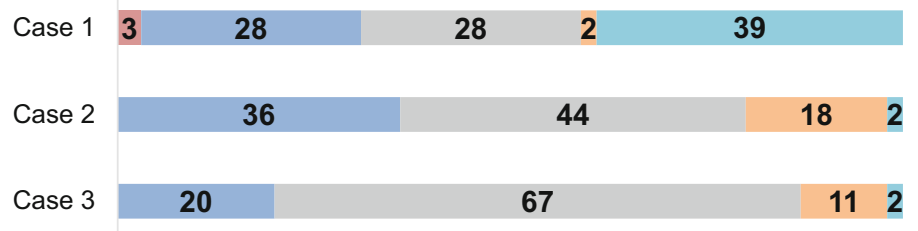

$\llbracket 56 \mathrm{~Gy} \backsim 60 \mathrm{~Gy} \backsim 64-66 \mathrm{~Gy} \backsim>66 \mathrm{~Gy} \backsim$ Question not answered

e Indication for CTX

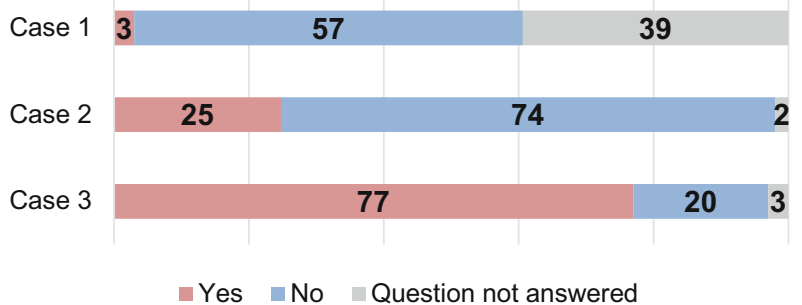

Fig. 3 Proposed treatment concepts for cases 1 to 3. a Indication for PORT (\%); b target volume definition of PTR (\%); $\mathbf{c}$ indication for nodal irradiation (\%); d dose prescription for PTR (\%); e indication for CTX (\%). PORT postoperative radiotherapy, PTR primary tumor region, CTX chemotherapy 
Fig. 4 Details on indications for treatment and scope of treatment for stereotactic ablative body radiotherapy of distant metastases. $S A B R$ stereotactic ablative body radiotherapy

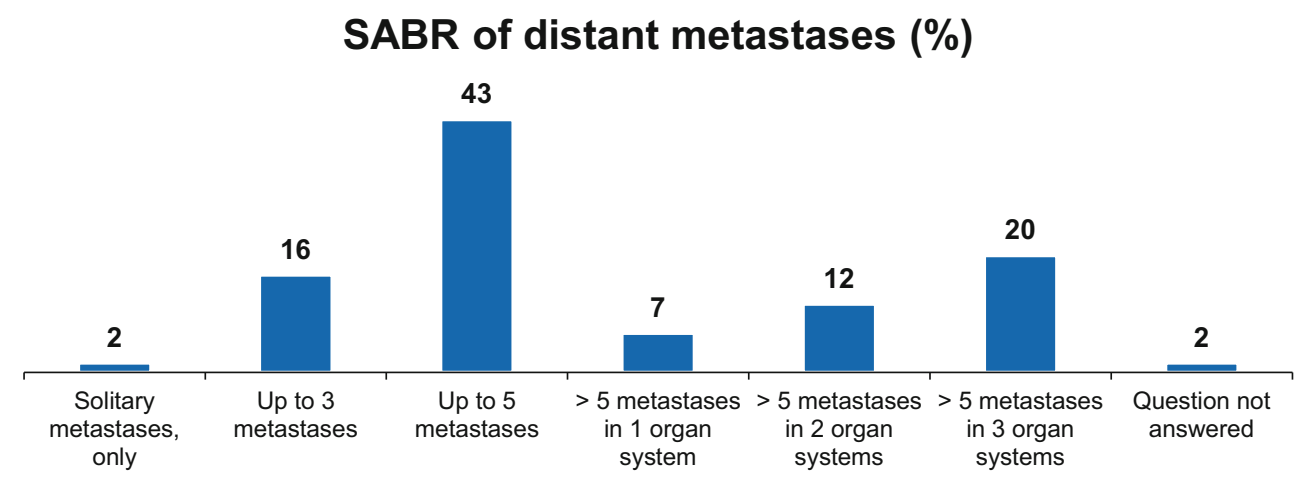

Case 5 presented a patient with locally controlled AdCC 3 years after primary therapy with first diagnosis of 2 lung and 2 bone metastases. The following treatment options were chosen: $29.5 \%$ CTX, 21.3\% immunotherapy (IT), 78.7\% stereotactic ablative body radiotherapy (SABR), $24.6 \%$ surgery, and $4.9 \%$ best supportive care (BSC). For details on indications for treatment and scope of treatment for SABR of distant metastases, see Fig. 4.

Comparing university vs. non-university institutions (including community hospitals and private practices), there was a significant difference in the number of patients treated per year and the extent of additional molecular pathological diagnostics. Regarding radiation techniques used, target volume definition, and concomitant CTX application, no significant differences between university and non-university institutions were found (Table 3).

\section{Discussion}

To the best of our knowledge, the present pattern of care survey for SGC is the first to be carried out among members of the German Society of Radiation Oncologists and among radiation oncologists in general. No prospective trials on RT of SGC have been completed to date. Therefore, we conducted this survey to provide an overview of commonly used diagnostics and treatment modalities applied within the DEGRO. The results are discussed and compared to current literature and guidelines below, with the aim of providing recommendations for practical daily routine.

\section{Diagnostic imaging for radiotherapy planning}

According to current guidelines, salivary gland tumors are locally staged by either contrast-enhanced CT or MRI [4]. Accordingly, the majority of participants recommended initial CT and/or MRI scans (75.8-88.5\%). While a prior meta-analysis considered both modalities to be equally effective [5], a more recent meta-analysis by Kong et al. indicated pooled sensitivities and specificities for CT to be 70 and $73 \%$ and for MRI to be 80 and $90 \%$, respectively.
The authors suggested MRI as the first-choice modality [6]. In addition, $18 \%$ of the participants recommended PET-CT for routine tumor staging. Guidelines recommend ${ }^{18} \mathrm{~F}-\mathrm{FDG}-$ PET-CT for nodal and distant metastases assessment due to its high sensitivity $[4,7]$. However, ${ }^{18} \mathrm{~F}$-FDG-PET-CT cannot safely distinguish malignant processes from benign neoplasms such as Whartin's tumors with high glucose uptake for example [8]. Furthermore, glucose uptake in AdCC is lower than in squamous cell carcinoma (SCC), or may be non-existent [9]. AdCC and SDC often overexpress PSMA (Prostate-specific membrane antigen), indicating a benefit from the use of PSMA PET-CT for these entities [10,11] rather than ${ }^{11} \mathrm{C}$-choline PET-CT [10]. For PORT, participants frequently recommended postoperative CT (45.2\%) or MRI $(43.5 \%)$. Indeed, if incomplete tumor resection is expected or pathologically proven, postoperative imaging can guide target volume delineation for adjuvant or additive RT [12]. Recently, an ASCO (American Society of Clinical Oncology) guideline for the management of SGC was published [13]. The authors recommend CT or MRI of the neck as first-choice staging modalities.

Take home message CT scans have an added value when involvement of the bone is suspected and diffusionweighted MRI scans to rule out perineural invasion and skull base involvement. There is a weak recommendation to use PET-CT in cases of locally advanced or high-grade SGC.

\section{Dose prescription and target volume delineation}

\section{Primary tumor region}

Early studies regarding definitive photon RT in SGC showed very low locoregional control rates, but the prescribed radiation doses (50 to $60 \mathrm{~Gy}$ ) were mostly insufficient by current standards $[14,15]$. Chen et al. reported improved locoregional control rates when doses were escalated to above $66 \mathrm{~Gy}$ [16]. In definitive photon RT of AdCC applying doses $>70 \mathrm{~Gy}$, local control rates after 5 and 10 years of $56 \%$ and $43 \%$ for all T-stages and of 
Table 3 Comparison of university vs. non-university institutions

\begin{tabular}{|c|c|c|c|}
\hline Characters & University $(n)$ & Non-university $(n)$ & $p$-value \\
\hline \multicolumn{4}{|l|}{ No. of patients per year $(n=67)$} \\
\hline 0 & 0 & 6 & \\
\hline $1-3$ & 2 & 14 & \\
\hline $3-5$ & 1 & 12 & \\
\hline $5-10$ & 6 & 12 & \\
\hline$>10$ & 12 & 2 & $<0.001$ \\
\hline \multicolumn{4}{|c|}{ Use of further molecular pathological diagnostics } \\
\hline Yes & 16 & 18 & \\
\hline No & 5 & 22 & 0.020 \\
\hline \multicolumn{4}{|l|}{ Radiation technique } \\
\hline \multicolumn{4}{|l|}{ Primary RT for AdCC } \\
\hline IMRT & 2 & 2 & \\
\hline Rotational IMRT & 5 & 22 & \\
\hline Treatment with protons & 0 & 0 & \\
\hline Treatment with carbon ions & 3 & 1 & \\
\hline Referral to carbon ion/proton therapy center & 11 & 15 & 0.069 \\
\hline \multicolumn{4}{|l|}{ PORT for AdCC } \\
\hline IMRT & 3 & 3 & \\
\hline Rotational IMRT & 13 & 30 & \\
\hline Treatment with protons & 0 & 0 & \\
\hline Treatment with carbon ions & 1 & 0 & \\
\hline Referral to carbon ion/proton therapy center & 4 & 7 & 0.407 \\
\hline \multicolumn{4}{|l|}{ Primary RT for non-AdCC } \\
\hline IMRT & 3 & 3 & \\
\hline Rotational IMRT & 13 & 35 & \\
\hline Treatment with protons & 1 & 0 & \\
\hline Treatment with carbon ions & 1 & 0 & \\
\hline Referral to carbon ion/proton therapy center & 3 & 2 & 0.133 \\
\hline \multicolumn{4}{|l|}{ PORT for non-AdCC } \\
\hline IMRT & 3 & 3 & \\
\hline Rotational IMRT & 16 & 36 & \\
\hline Treatment with protons & 0 & 0 & \\
\hline Treatment with carbon ions & 1 & 0 & \\
\hline Referral to carbon ion/proton therapy center & 1 & 1 & 0.381 \\
\hline \multicolumn{4}{|l|}{ Use of concomitant CTX for PORT } \\
\hline Yes & 15 & 33 & \\
\hline No & 6 & 7 & 0.316 \\
\hline \multicolumn{4}{|l|}{ Use of concomitant CTX for primary RT } \\
\hline Yes & 11 & 26 & \\
\hline No & 10 & 14 & 0.338 \\
\hline \multicolumn{4}{|c|}{ Indications for irradiation of nerve tracts up to the base of skull } \\
\hline Never & 1 & 7 & \\
\hline Parotid gland carcinoma with Pn1 & 6 & 6 & \\
\hline All SGC with Pn1 & 6 & 10 & \\
\hline All AdCC with Pn1 & 3 & 8 & \\
\hline Any AdCC & 5 & 8 & 0.560 \\
\hline
\end{tabular}

$R T$ radiotherapy, PORT postoperative radiotherapy, IMRT intensity-modulated radiotherapy, AdCC adenoid cystic carcinoma, $C T X$ chemotherapy, $S G C$ salivary gland cancer 
$44 \%$ and $30 \%$ in T4 tumors only were reported, respectively [17]. According to ASCO guidelines, for patients with unresectable disease, the primary tumor and gross nodal disease should be treated with 70Gy in 2-Gy fractions [13]. Regarding the former primary tumor region for PORT, a minimum dose of $60 \mathrm{~Gy}$ following complete tumor resection and $66 \mathrm{~Gy}$ in case of positive resection margins have been recommended before [1]. Indeed, most participants chose doses between 60 and $66 \mathrm{~Gy}$ for PORT. The American guidelines also recommend $\geq 60$ Gy in $2-G y$ fractions to the former primary tumor region in terms of the salivary gland surgical bed and to appropriate nodal levels [13].

Take home message In case of macroscopic disease, a dose of $>70 \mathrm{~Gy}$ should be applied in the tumor region. In the postoperative situation, dose prescription ranges from 60 to $66 \mathrm{~Gy}$ according to risk factors such as microscopic residual disease and/or perineural spread.

\section{Elective nodal irradiation}

The following recommendations concerning target volume definition in SGC have been proposed:

Clinical target volume (CTV) 1 should include the macroscopic tumor and tumor bed while CTV2 includes CTV1 plus high-risk and ipsilateral nodal levels. If the primary tumor crosses the midline, bilateral nodes should be included [18]. Several guidelines recommend elective nodal irradiation in cN0 patients as well as elective nodal coverage in case of locally advanced T-stage T3/T4 and in high-grade tumors. However, the histological subtype should be considered. The risk of lymph node metastases is higher in undifferentiated, adeno-, and mucoepidermoid carcinoma compared to AdCC and AciCC [19]. Regarding PORT, recommendations for dose prescription vary between 44, 46, and 50 Gy for the elective CTV2 [4, 13].

Take home message The presence of lymph node metastasis depends on tumor histology, T-stage, and grading. Elective nodal irradiation should be individually discussed and applied in case of high-grade histology and advanced T-stage, especially in undifferentiated, adeno-, salivary duct, and mucoepidermoid carcinoma.

\section{Radiation of nerve tracts up to the base of skull}

The opinions of the participants with respect to target volume definition, especially regarding the inclusion of the nerve tracts up to the base of skull and irradiation of elective neck nodes, varied greatly.

Perineural spread is a common finding in SGC, especially AdCC. In case of perineural spread, the target vol- ume should include the relevant cranial nerve pathways at risk [20]. According to the literature, SGC recurrences to the base of skull were reduced by PORT $[13,21]$. Recommended doses for the involved nerve vary from 46 to $54 \mathrm{~Gy}$ [13].

Take home message In case of perineural spread, the target volume should include the nerve tracts up to the base of skull.

\section{Radiation treatment technique}

Overall, the preferred treatment technique was IMRT in concordance with the literature [13]. In case of treating AdCC in the primary situation, $50 \%$ of the participants would prefer carbon ion RT. Proportions were lower for PORT and non-AdCC. No prospective trials comparing photon with proton or carbon ion radiotherapy exist. Nonetheless, most experience with carbon ion therapy in SGC comes from the treatment of AdCC. A retrospective trial reported superior 5-year locoregional control in the treatment of AdCC using carbon ion instead of photon RT (60 vs. 40\%) [22]. Retrospective long-term data including over 300 patients showed 5-year locoregional control rates of $58 \%$ in all patients and 70.9 and $38.6 \%$ in T4a and T4b tumors, respectively [3]. In SGC other than AdCC, the value of carbon ions or protons remains even more unclear. Regarding the combination of IMRT with carbon ion boost, the phase II COSMIC trial [18] enrolled postoperative SGC patients with positive resection margins and/or perineural spread or primarily inoperable patients. Initial results reported an overall locoregional control rate of $81.9 \%$ for all patients included and of $89.7 \%$ for patients with microscopic incomplete resection margin and/or perineural spread after 3 years. However, local control rates were similar to those reported with photon RT $[1,23]$.

Take home message According to these data, particle therapy may be used in SGC. Because prospective comparisons with IMRT are scarce, particle therapy remains without clear added value over modern photon therapy so far.

\section{Concomitant chemoradiotherapy}

Twenty-one percent of the participants never recommend CTX for primary RT and nearly $40 \%$ for PORT. When CTX is prescribed, it is usually based on an individual decision (60\%). Indeed, concomitant RCT is a controversial topic in the treatment of SGC, since hardly any data exist to this regard. A retrospective database analysis could not show any benefit regarding overall survival (OS) for the combination of postoperative RT and CTX [24] and is in line with prior investigations [25]. Unfor- 
tunately, high-grade SGC mostly show distant metastases as the first recurrence, which possibly relativizes the potential benefit of an intensified local therapy regarding OS $[18,26]$. In matched-pair analyses and small monoinstitutional studies, improved locoregional control and/or better progression-free survival in patients receiving platinbased chemotherapy has been reported for SGC [27-29]. Results from a prospective, randomized RTOG 1008 trial (NCT01220583) investigating postoperative radiotherapy with or without weekly cisplatin in high-risk SGC and two other similar trials (NCT02776163, NCT02998385) have not been reported so far. But regarding the fact that radiotherapy leads to high locoregional control rates and most common failures are distant, it has to be assumed that progression-free survival may not be significantly increased in ongoing trials. However, as case reports $1-3$ in this study show, simultaneously applied chemotherapy is performed in daily routine in many centers and its implementation correlates with increasing risk factors. Interestingly, in case 3, 13 participants would apply a trastuzumab-based therapy in the postoperative situation. Indeed, some retrospective case series demonstrate improved disease-free survival (DFS) and OS in patients receiving trastuzumab-based therapy or androgen deprivation in the postoperative setting [30, 31]. This targeted therapy may be considered in patients with salivary duct carcinoma with androgen receptor expression and HER2neu amplification according to individual risk factors. However, it remains unclear whether these forms of systemic treatment provide any benefit when applied simultaneously to RT and if such combined regimens should be routinely used. According to the new ASCO guideline, concurrent CTX should not be routinely offered outside clinical trials, whether in the definitive or in the postoperative setting [13].

Take home message Results of prospective randomized trials investigating combined CRT in SGC are pending. Concomitant CTX should not be offered routinely.

\section{Molecular diagnostics}

Within this survey, the most commonly performed molecular diagnostics were HER2neu amplification, PD-L1 status, and AR status. While the current recommendations for molecular diagnostics with regards to the primary tumor diagnosis are weak, there are indeed several clearer recommendations when systemic therapy is planned: $A R$ in SDC and NTRK fusion in MASC; AR, HER2neu, and NTRK fusion may be offered for non-AdCC; tumor mutational burden (TMB), microsatellite instability (MSI) prior to checkpoint inhibition; next-generation sequencing (NGS) for tumor types with low prevalence. Interestingly, no rec- ommendations regarding the clinical value of the PD-L1 status exist yet [13].

Take home message There is no clear value of comprehensive molecular diagnostics in the primary situation. In case of metastatic disease, molecular diagnostics according to tumor subtype should be performed to evaluate possible targeted therapies. Moreover TMB, MSI, or NGS should be considered.

\section{Discussion of case presentations}

Cases 1 and 2 presented AdCC in different tumor stages following complete resection. AdCC frequently metastasizes, even in cases of early primary tumors. Therefore, controversy exists about PORT for pT1-2N0 tumors to minimize the risk of distant progression, while PORT is strongly recommended for any more advanced tumors [32]. Current guidelines suggest PORT (evidence category 2B) in SGC for intermediate- or high-grade tumors, close or positive margins, perineural invasion, lymph node metastases, lymphovascular invasion, T3-4 tumors, or any AdCC [4]. However, a National Cancer Database (NCDB) analysis encompassing 1784 AdCC patients found survival differences clearly favoring postoperative irradiation also for the pT12N0 subgroup [33]. In contrast, two large and stage-independent analyses from the US American Surveillance, Epidemiology, and End Results Database (SEER) found no benefit for PORT for this cohort [34, 35]. Furthermore, following analysis of the benefit of PORT on local tumor control after adjustment for T-stage, Ali et al. recommended PORT for all AdCC patients, possibly with the exception of small T1 tumors without adverse features [36]. PORT for all AdCC is also strongly recommended by the current ASCO guidelines [13]. For targeting the primary tumor region, radiation doses of over $60 \mathrm{~Gy}$ for AdCC were recommended by Chen et al. [16].

Take home message PORT is recommended for any adenoid cystic carcinoma.

Case 4 presented a T4, low-grade AciCC with positive resection margins. Most participants decided for PORT. As described above, T4 stage and positive resection margins indicate PORT according to the guidelines and a number of authors [4, 37-40]. Since incomplete excisions were associated with impaired survival, PORT should be considered [39]. However, a SEER database analysis including 1241 cases of AciCC from the parotid gland found no survival advantage for early-stage or low-grade tumors and results for highest-stage and highest-grade tumors were inconclusive [41]. Nevertheless, it has to be considered that this analysis was retrospective and without detailed patient characteristics of the two treatment groups (surgery $+\mathrm{RT}$ vs. 
surgery alone). It has to be assumed that patients undergoing radiotherapy had more pathological risk factors. Taken together, giving a clear postoperative treatment recommendation for this scenario remains difficult and individual factors should also be taken into account in a multidisciplinary tumor board.

Case 5 tried to elucidate patterns of care in the oligometastatic situation. Patients with SGC presenting in this disease stage are often treated with individual concepts, mainly due to the lack of effective systemic treatment alternatives and a relatively young age. One of these approaches is metastasis-directed, locally radical therapy via surgery or SABR. Earlier retrospective data demonstrate 5-year OS rates of 20-54\% for the combination of high-intensity metastasisdirected local therapy (i.e. surgery or SABR) and systemic treatment for various entities of oligometastatic head and neck cancer [42, 43]. Advances in RT and especially SABR [44-46] allowed an improvement in disease control and/or survival in different tumor entities in the oligometastatic setting [47-49]. Thus, adding local treatment in this situation appears to be promising. Especially in the case of SABR, this strategy is also based on a strong biological rationale: SABR has well-investigated immunosensitizing features [50]. A large, disease agnostic, randomized trial could recently prove the significant benefit of such an approach for 1-5 metastases when metastasis-directed SABR was added to standard of care for various malignancies [51]. Furthermore, in a subsequent trial by the same group, open for recruitment, this concept is currently being prospectively evaluated even for 4-10 metastases [52]. In the case of SGC, where slowly growing metastases are often observed for some histologic subtypes, metastasisdirected SABR could be a useful tool providing excellent control rates in various organs $[53,54]$. A better understanding of the different clinical situations, like oligoprogression and oligopersistence [55], and abandoning the arbitrary oligometastatic definition based solely on the number of metastases, will allow for even better patient selection and oncological results in the future. Moreover, for oligometastatic SGC, the individual histology and tumor grading should also be considered. The current guidelines offer a recommendation in the recurrent and/or metastatic (R/M) setting to evaluate SABR as an option besides systemic therapy. Further, for R/M AdCC and low-grade tumors, SABR may be offered for a limited number of metastases $(\leq 5)[13]$.

Take home message In oligometastatic disease, SABR and other local therapeutic strategies should be evaluated considering individual histology, progression of disease, and systemic treatment options.

Six participants from private practices and non-university hospitals reported not to treat any SGC at all at their facili- ties. Depending on histological subtype and the therapeutic setting, up to $43 \%$ of the participants would present their patients at a proton/carbon ion tumor center. The treatment of SGC poses a major challenge to any radiation oncologist. Besides the inherent radioresistance of SGC, also the complex shaping of target volumes in combination with their proximity to radiosensitive organs at risk hampers treatment planning [56]. Indeed, all participants would refrain from 3D-RT in favor of more advanced RT techniques as recommended in the literature [56]. Intriguingly, a recent analysis within the NCDB revealed no OS benefit for patients treated at high-volume facilities (HVF) or academic/ research institutions. However, patients treated at HVF had more secondary diseases and advanced tumor stages [57]. In order to facilitate the collection of prospective data on the different SGC subtypes and to provide future guidelines, especially considering individual (radiation) treatment options in the different histologic subtypes, the accumulation of patients at certain HVF could be beneficial.

\section{Conclusion}

SGC represents a major challenge for clinicians, as reflected by the inhomogeneous survey results regarding diagnostics, RT, and systemic therapy. The difficulties for practitioners arise mainly from the large number of existing and often rare subtypes with different biological behavior and aggressiveness and the lack of high-level evidence. Future prospective, clinical trials are warranted to improve and homogenize treatment of SGC, and especially to give recommendations for the individual tumor subtypes. Large prospective registers could help to overcome the issues of rarity and heterogeneity of the diagnosis.

Supplementary Information The online version of this article (https:// doi.org/10.1007/s00066-021-01833-x) contains supplementary material, which is available to authorized users.

Acknowledgements This work was supported by grants from the Else Kröner-Fresenius-Stiftung to Jens von der Grün.

Funding The authors did not receive support from any organization for the submitted work.

Funding Open Access funding enabled and organized by Projekt DEAL.

Conflict of interest J. von der Grün, C. Rödel, S. Semrau, P. Balermpas, D. Martin, R. Fietkau, and M. Haderlein declare that they have no competing interests.

Open Access This article is licensed under a Creative Commons Attribution 4.0 International License, which permits use, sharing, adaptation, distribution and reproduction in any medium or format, as long as you give appropriate credit to the original author(s) and the source, provide a link to the Creative Commons licence, and indicate if changes were made. The images or other third party material in this article are 
included in the article's Creative Commons licence, unless indicated otherwise in a credit line to the material. If material is not included in the article's Creative Commons licence and your intended use is not permitted by statutory regulation or exceeds the permitted use, you will need to obtain permission directly from the copyright holder. To view a copy of this licence, visit http://creativecommons.org/licenses/by/4. $0 /$.

\section{References}

1. Garden AS, Weber RS, Morrison WH, Ang KK, Peters LJ (1995) The influence of positive margins and nerve invasion in adenoid cystic carcinoma of the head and neck treated with surgery and radiation. Int J Radiat Oncol Biol Phys 32(3):619-626. https://doi. org/10.1016/0360-3016(95)00122-F

2. Terhaard CH, Lubsen H, Rasch CR, Levendag PC, Kaanders HH, Tjho-Heslinga RE et al (2005) The role of radiotherapy in the treatment of malignant salivary gland tumors. Int J Radiat Oncol Biol Phys 61(1):103-111. https://doi.org/10.1016/j.ijrobp.2004.03.018

3. Jensen AD, Poulakis M, Nikoghosyan AV, Welzel T, Uhl M, Federspil PA et al (2016) High-LET radiotherapy for adenoid cystic carcinoma of the head and neck: 15 years' experience with rasterscanned carbon ion therapy. Radiother Oncol 118(2):272-280. https://doi.org/10.1016/j.radonc.2015.05.010

4. National Comprehensive Cancer Network (2020) Head and neck cancers (Version 2.2020, June 09, 2020). https://www.nccn.org/ professionals/physician_gls/pdf/head-and-neck.pdf. Accessed 20 Oct 2020

5. Liu Y, Li J, Tan YR, Xiong P, Zhong LP (2015) Accuracy of diagnosis of salivary gland tumors with the use of ultrasonography, computed tomography, and magnetic resonance imaging: a meta-analysis. Oral Surg Oral Med Oral Pathol Oral Radiol 119(2):238-245.e2. https://doi.org/10.1016/j.oooo.2014.10.020

6. Kong X, Li H, Han Z (2019) The diagnostic role of ultrasonography, computed tomography, magnetic resonance imaging, positron emission tomography/computed tomography, and real-time elastography in the differentiation of benign and malignant salivary gland tumors: a meta-analysis. Oral Surg Oral Med Oral Pathol Oral Radiol 128(4):431-443.e1. https://doi.org/10.1016/j.oooo. 2019.06.014

7. Freling N, Crippa F, Maroldi R (2016) Staging and follow-up of high-grade malignant salivary gland tumours: the role of traditional versus functional imaging approaches-A review. Oral Oncol 60:157-166. https://doi.org/10.1016/j.oraloncology.2016.04.016

8. Park HL, Yoo Ie R, Lee N, Yoon H, Choi EK, Choi HS et al (2013) The value of F-18 FDG PET for planning treatment and detecting recurrence in malignant salivary gland tumors: comparison with conventional imaging studies. Nucl Med Mol Imaging 47(4):242-248. https://doi.org/10.1007/s13139-013-0222-8

9. Jung JH, Lee SW, Son SH, Kim CY, Lee CH, Jeong JH et al (2017) Clinical impact of (18) F-FDG positron emission tomography/CT on adenoid cystic carcinoma of the head and neck. Head Neck 39(3):447-455. https://doi.org/10.1002/hed.24605

10. van Boxtel W, Lutje S, van Engen-van Grunsven ICH, Verhaegh GW, Schalken JA, Jonker MA et al (2020) (68)Ga-PSMAHBED-CC PET/CT imaging for adenoid cystic carcinoma and salivary duct carcinoma: a phase 2 imaging study. Theranostics 10(5):2273-2283. https://doi.org/10.7150/thno.38501

11. Klein Nulent TJW, van Es RJJ, Krijger GC, de Bree R, Willems SM, de Keizer B (2017) Prostate-specific membrane antigen PET imaging and immunohistochemistry in adenoid cystic carcinoma-A preliminary analysis. Eur J Nucl Med Mol Imaging 44(10):1614-1621. https://doi.org/10.1007/s00259-017-3737-x

12. Di L, Qian K, Du C, Shen C, Zhai R, He X et al (2018) Radiotherapy as salvage treatment of salivary duct carcinoma in major salivary glands without radical operations. Cancer Manag Res 10:6071-6078. https://doi.org/10.2147/CMAR.S185542

13. Geiger JL, Ismaila N, Beadle B, Caudell JJ, Chau N, Deschler D et al (2021) Management of salivary gland malignancy: ASCO guideline. J Clin Oncol 39(17):1909-1941. https://doi.org/10.1200/ JCO.21.00449

14. Vikram B, Strong EW, Shah JP, Spiro RH (1984) Radiation therapy in adenoid-cystic carcinoma. Int $\mathrm{J}$ Radiat Oncol Biol Phys 10(2):221-223

15. Fitzpatrick PJ, Theriault C (1986) Malignant salivary gland tumors. Int J Radiat Oncol Biol Phys 12(10):1743-1747

16. Chen AM, Bucci MK, Weinberg V, Garcia J, Quivey JM, Schechter NR et al (2006) Adenoid cystic carcinoma of the head and neck treated by surgery with or without postoperative radiation therapy: prognostic features of recurrence. Int J Radiat Oncol Biol Phys 66(1):152-159. https://doi.org/10.1016/j.ijrobp.2006.04.014

17. Mendenhall WM, Morris CG, Amdur RJ, Werning JW, Hinerman RW, Villaret DB (2004) Radiotherapy alone or combined with surgery for adenoid cystic carcinoma of the head and neck. Head Neck 26(2):154-162. https://doi.org/10.1002/hed.10380

18. Jensen AD, Nikoghosyan AV, Lossner K, Haberer T, Jakel O, Munter MW et al (2015) COSMIC: a regimen of intensity modulated radiation therapy plus dose-escalated, raster-scanned carbon Ion boost for malignant salivary gland tumors: results of the prospective phase 2 trial. Int J Radiat Oncol Biol Phys 93(1):37-46. https://doi.org/10.1016/j.ijrobp.2015.05.013

19. Chen AM, Garcia J, Lee NY, Bucci MK, Eisele DW (2007) Patterns of nodal relapse after surgery and postoperative radiation therapy for carcinomas of the major and minor salivary glands: what is the role of elective neck irradiation? Int J Radiat Oncol Biol Phys 67(4):988-994. https://doi.org/10.1016/j.ijrobp.2006.10.044

20. Armstrong K, Ward J, Hughes NM, Mihai A, Blayney A, Mascott $\mathrm{C}$ et al (2018) Guidelines for clinical target volume definition for perineural spread of major salivary gland cancers. Clin Oncol ( R Coll Radiol) 30(12):773-779. https://doi.org/10.1016/j.clon.2018. 08.018

21. Chen AM, Garcia J, Granchi P, Bucci MK, Lee NY (2009) Base of skull recurrences after treatment of salivary gland cancer with perineural invasion reduced by postoperative radiotherapy. Clin Otolaryngol 34(6):539-545. https://doi.org/10.1111/j.1749-4486.2009. 02036.x

22. Jensen AD, Nikoghosyan AV, Poulakis M, Hoss A, Haberer T, Jakel O et al (2015) Combined intensity-modulated radiotherapy plus raster-scanned carbon ion boost for advanced adenoid cystic carcinoma of the head and neck results in superior locoregional control and overall survival. Cancer 121(17):3001-3009. https:// doi.org/10.1002/cncr.29443

23. Haderlein M, Scherl C, Semrau S, Lettmaier S, Hecht M, Putz F et al (2019) Salivary gland carcinoma (SGC) with perineural spread and/or positive resection margin-High locoregional control rates after photon (chemo) radiotherapy-Experience from a monocentric analysis. Radiat Oncol 14(1):68. https://doi.org/10.1186/ s13014-019-1260-x

24. Amini A, Waxweiler TV, Brower JV, Jones BL, McDermott JD, Raben D et al (2016) Association of adjuvant chemoradiotherapy vs radiotherapy alone with survival in patients with resected major salivary gland carcinoma: data from the National Cancer Data base. JAMA Otolaryngol Head Neck Surg 142(11):1100-1110. https:// doi.org/10.1001/jamaoto.2016.2168

25. Tanvetyanon T, Fisher K, Caudell J, Otto K, Padhya T, Trotti A (2016) Adjuvant chemoradiotherapy versus with radiotherapy alone for locally advanced salivary gland carcinoma among older patients. Head Neck 38(6):863-870. https://doi.org/10.1002/hed. 24172

26. Terhaard $\mathrm{CH}$, Lubsen H, Van der Tweel I, Hilgers FJ, Eijkenboom WM, Marres HA et al (2004) Salivary gland carcinoma: 
independent prognostic factors for locoregional control, distant metastases, and overall survival: results of the Dutch head and neck oncology cooperative group. Head Neck 26(8):681-692. https:// doi.org/10.1002/hed.10400 (discussion 92-93)

27. Tanvetyanon T, Qin D, Padhya T, McCaffrey J, Zhu W, Boulware D et al (2009) Outcomes of postoperative concurrent chemoradiotherapy for locally advanced major salivary gland carcinoma. Arch Otolaryngol Head Neck Surg 135(7):687-692. https://doi.org/10.1001/ archoto. 2009.70

28. Schoenfeld JD, Sher DJ, Norris CM Jr., Haddad RI, Posner MR, Balboni TA et al (2012) Salivary gland tumors treated with adjuvant intensity-modulated radiotherapy with or without concurrent chemotherapy. Int J Radiat Oncol Biol Phys 82(1):308-314. https:// doi.org/10.1016/j.ijrobp.2010.09.042

29. Haderlein M, Scherl C, Semrau S, Lettmaier S, Uter W, Neukam FW et al (2016) High-grade histology as predictor of early distant metastases and decreased disease-free survival in salivary gland cancer irrespective of tumor subtype. Head Neck 38(Suppl 1):E2041-E2048. https://doi.org/10.1002/hed.24375

30. Hanna GJ, Bae JE, Lorch JH, Haddad RI, Jo VY, Schoenfeld JD et al (2020) The benefits of adjuvant trastuzumab for HER-2-positive salivary gland cancers. Oncologist. https://doi.org/10.1634/ theoncologist.2019-0841

31. van Boxtel W, Locati LD, van Engen-van Grunsven ACH, Bergamini C, Jonker MA, Fiets E et al (2019) Adjuvant androgen deprivation therapy for poor-risk, androgen receptor-positive salivary duct carcinoma. Eur J Cancer 110:62-70. https://doi.org/10.1016/j.ejca. 2018.12.035

32. Garg M, Tudor-Green B, Bisase B (2019) Current thinking in the management of adenoid cystic carcinoma of the head and neck. Br J Oral Maxillofac Surg 57(8):716-721. https://doi.org/10.1016/ j.bjoms.2019.07.021

33. Lee A, Givi B, Osborn VW, Schwartz D, Schreiber D (2017) Patterns of care and survival of adjuvant radiation for major salivary adenoid cystic carcinoma. Laryngoscope 127(9):2057-2062. https://doi.org/10.1002/lary.26516

34. Ellington CL, Goodman M, Kono SA, Grist W, Wadsworth T, Chen AY et al (2012) Adenoid cystic carcinoma of the head and neck: incidence and survival trends based on 1973-2007 surveillance, epidemiology, and end results data. Cancer 118(18):4444-4451. https://doi.org/10.1002/cncr.27408

35. Lloyd S, Yu JB, Wilson LD, Decker RH (2011) Determinants and patterns of survival in adenoid cystic carcinoma of the head and neck, including an analysis of adjuvant radiation therapy. Am J Clin Oncol 34(1):76-81. https://doi.org/10.1097/COC. 0b013e3181d26d45

36. Ali S, Palmer FL, Katabi N, Lee N, Shah JP, Patel SG et al (2017) Long-term local control rates of patients with adenoid cystic carcinoma of the head and neck managed by surgery and postoperative radiation. Laryngoscope 127(10):2265-2269. https://doi.org/ 10.1002/lary.26565

37. Greig SR, Chaplin JM, McIvor NP, Izzard ME, Taylor G, Wee D (2008) Acinic cell carcinoma of the parotid gland: Auckland experience and literature review. ANZ J Surg 78(9):754-758. https:// doi.org/10.1111/j.1445-2197.2008.04643.x

38. Spafford PD, Mintz DR, Hay J (1991) Acinic cell carcinoma of the parotid gland: review and management. J Otolaryngol 20(4):262-266

39. Al-Zaher N, Obeid A, Al-Salam S, Al-Kayyali BS (2009) Acinic cell carcinoma of the salivary glands: a literature review. Hematol Oncol Stem Cell Ther 2(1):259-264. https://doi.org/10.1016/ s1658-3876(09)50035-0

40. Cavaliere M, De Luca P, Scarpa A, Savignano L, Cassandro C, Cassandro E et al (2020) Acinic cell carcinoma of the parotid gland: from pathogenesis to management: a literature review. Eur
Arch Otorhinolaryngol 277(10):2673-2679. https://doi.org/10. 1007/s00405-020-05998-6

41. Andreoli MT, Andreoli SM, Shrime MG, Devaiah AK (2012) Radiotherapy in parotid acinic cell carcinoma: does it have an impact on survival? Arch Otolaryngol Head Neck Surg 138(5):463-466. https://doi.org/10.1001/archoto.2012.226

42. Sun XS, Michel C, Babin E, De Raucourt D, Pechery A, Gherga E et al (2018) Approach to oligometastatic disease in head and neck cancer, on behalf of the GORTEC. Future Oncol 14(9):877-889. https://doi.org/10.2217/fon-2017-0468

43. Thomas TVV, Packianathan S, Bhanat E, Albert AA, Abraham A, Gordy X et al (2019) Management of oligometastatic squamous cell carcinoma of the head and neck. Int J Radiat Oncol Biol Phys 104(5):1191. https://doi.org/10.1016/j.ijrobp.2019.05.048

44. Guckenberger M, Baus WW, Blanck O, Combs SE, Debus J, Engenhart-Cabillic R et al (2020) Definition and quality requirements for stereotactic radiotherapy: consensus statement from the DEGRO/DGMP working group stereotactic radiotherapy and radiosurgery. Strahlenther Onkol 196(5):417-420. https://doi.org/10. 1007/s00066-020-01603-1

45. Schmitt D, Blanck O, Gauer T, Fix MK, Brunner TB, Fleckenstein $\mathrm{J}$ et al (2020) Technological quality requirements for stereotactic radiotherapy: expert review group consensus from the DGMP working group for physics and technology in stereotactic radiotherapy. Strahlenther Onkol 196(5):421-443. https://doi.org/10.1007/ s00066-020-01583-2

46. Wilke L, Andratschke N, Blanck O, Brunner TB, Combs SE, Grosu AL et al (2019) ICRU report 91 on prescribing, recording, and reporting of stereotactic treatments with small photon beams: statement from the DEGRO/DGMP working group stereotactic radiotherapy and radiosurgery. Strahlenther Onkol 195(3):193-198. https://doi.org/10.1007/s00066-018-1416-x

47. Gomez DR, Tang C, Zhang J, Blumenschein GR Jr., Hernandez M, Lee JJ et al (2019) Local consolidative therapy vs. maintenance therapy or observation for patients with oligometastatic non-smallcell lung cancer: long-term results of a multi-institutional, phase II, randomized study. J Clin Oncol 37(18):1558-1565. https://doi.org/ 10.1200/JCO.19.00201

48. Iyengar P, Wardak Z, Gerber DE, Tumati V, Ahn C, Hughes RS et al (2018) Consolidative radiotherapy for limited metastatic nonsmall-cell lung cancer: a phase 2 randomized clinical trial. JAMA Oncol 4(1):e173501. https://doi.org/10.1001/jamaoncol.2017.3501

49. Parker CC, James ND, Brawley CD, Clarke NW, Hoyle AP, Ali A et al (2018) Radiotherapy to the primary tumour for newly diagnosed, metastatic prostate cancer (STAMPEDE): a randomised controlled phase 3 trial. Lancet 392(10162):2353-2366. https://doi. org/10.1016/S0140-6736(18)32486-3

50. Sharabi AB, Lim M, DeWeese TL, Drake CG (2015) Radiation and checkpoint blockade immunotherapy: radiosensitisation and potential mechanisms of synergy. Lancet Oncol 16(13):e498-e509. https://doi.org/10.1016/S1470-2045(15)00007-8

51. Palma DA, Olson R, Harrow S, Gaede S, Louie AV, Haasbeek C et al (2020) Stereotactic ablative radiotherapy for the comprehensive treatment of oligometastatic cancers: long-term results of the SABR-COMET phase II randomized trial. J Clin Oncol 38(25):2830-2838. https://doi.org/10.1200/JCO.20.00818

52. Palma DA, Olson R, Harrow S, Correa RJM, Schneiders F, Haasbeek CJA et al (2019) Stereotactic ablative radiotherapy for the comprehensive treatment of 4-10 oligometastatic tumors (SABRCOMET-10): study protocol for a randomized phase III trial. BMC Cancer 19(1):816. https://doi.org/10.1186/s12885-019-5977-6

53. Klement RJ, Hoerner-Rieber J, Adebahr S, Andratschke N, Blanck O, Boda-Heggemann J et al (2018) Stereotactic body radiotherapy (SBRT) for multiple pulmonary oligometastases: analysis of number and timing of repeat SBRT as impact factors on treatment safety 
and efficacy. Radiother Oncol 127(2):246-252. https://doi.org/10. 1016/j.radonc.2018.02.016

54. Andratschke N, Alheid H, Allgäuer M, Becker G, Blanck O, BodaHeggemann J et al (2018) The SBRT database initiative of the German Society for Radiation Oncology (DEGRO): patterns of care and outcome analysis of stereotactic body radiotherapy (SBRT) for liver oligometastases in 474 patients with 623 metastases. BMC Cancer 18(1):283. https://doi.org/10.1186/s12885-018-4191-2

55. Guckenberger M, Lievens Y, Bouma AB, Collette L, Dekker A, deSouza NM et al (2020) Characterisation and classification of oligometastatic disease: a European society for radiotherapy and oncology and European organisation for research and treatment of cancer consensus recommendation. Lancet Oncol 21(1):e18-e28. https://doi.org/10.1016/S1470-2045(19)30718-1

56. Orlandi E, Iacovelli NA, Bonora M, Cavallo A, Fossati P (2016) Salivary gland. Photon beam and particle radiotherapy: present and future. Oral Oncol 60:146-156. https://doi.org/10.1016/j. oraloncology.2016.06.019

57. Bollig CA, Zitsch RP 3rd (2019) Impact of treating facilities' type and volume in patients with major salivary gland cancer. Laryngoscope 129(10):2321-2327. https://doi.org/10.1002/lary.27844 